\title{
Crashworthiness analysis of octagonal multi-cell tube with functionally graded thickness under multiple loading angles
}

\author{
Yafeng Chen, Zhonghao Bai", Linwei Zhang, Yulong Wang, Guangyong Sun, Libo Cao
}

\begin{abstract}
:
This paper provides a study on a novel octagonal multi-cell tube with functionally graded thickness (FGT) under multiple loading angles. First, comparative analysis on the FGT tube and the counterpart tube with uniform thickness(UT) under multiple loading angles reveal that the energy absorption is more superior for the FGT tube when the loading angle exceeds the lower bound of the transition range of the UT tube. Second, parametric study on the FGT tube indicates that thickness gradient exponent and thickness range have significant effect on its crashworthiness. Third, multiobjective optimizations of the FGT tube are conducted, aiming to maximize specific energy absorption(SEA) and minimize initial force(IPF) under multiple loading angles, based upon the Non-dominated sorting genetic algorithm(NAGA- II ) and RBF technique. The optimized FGT tube demonstrates better crashworthiness than the UT tube in all design cases. These findings can provide valuable guidelines for the design of multi-cell tube with functionally graded thickness under multiple loading angles.
\end{abstract}

\section{Keywords:}

Octagonal multi-cell tube; functionally graded thickness; multiple oblique impact; crashworthiness optimization. 


\section{Introduction}

To reduce the risk of occupant injury during the car crash accidents, the vehicles have to be designed with good crashworthiness, to maintain a low level acceleration and small intrusion of the occupant compartment. To achieve these goals, one approach is to use energy absorbing structures, which can dissipate crash energy through large plastic deformation and tearing damage. In the past decades, there are a large amount experimental, theoretical and computational studies conducted on different types of energy absorbers[1-13]. For example, comprehensive research work has been performed to investigate the effect of cross-sectional shape of a thin-walled structure (e.g. circular[1-4], square, tapered and polygonal[5-13]) on its crashworthy performance. It has been reported that under axial loading, octagonal tube has a better performance than its square and hexagonal counterparts[11]. When the number of edges exceeds 8 , the mean force and energy efficiency become convergent[12]. In addition, compared to the tubes with odd number of edges, those with even numbers of edges exhibit a more regular folding mode[13]. The energy absorbing capability of a single cell tube is limited by its edges. To improve its performance, people consider adding inner blades to form a multiple cell structure[14-19]. Zhang et al.[14] compared the energy absorber efficiencies of aluminum alloy by testing single and multiple cell square tubes under axial impact. The results show that the performance of multiple cell structure is higher than that of the single cell tube by 50 100\%. Annisa et al.[15]conducted numerical analysis on several kinds of multi-cell prismatic structures and found that the energy absorption efficiency could be significantly improved by introducing internal ribs to the double-walled columns. Nia et al. [16]compared triangular, square, hexagonal and octagonal single and multiple cell tubes under quasi-static axial loading. It is found that a type of octagonal multi-cell tube with inner blades connected demonstrated the best mean force and specific energy.

These above-mentioned studies are focused on the axial loading conditions. But in reality, tubular energy absorbers are not likely subjected to uniaxial impact only. The work by Han et al.[20]suggested that the angle of loading could directly change the deformation mode of the structure. There existed a critical or threshold angle, which was a transition between 
progressive collapse and lateral bending modes. Hosseini-Tehrani and Pirmohammad[21]investgated the effect of loading angle on mean crush loads and permanent displacements for square, hexagonal, octagonal and decagonal tubes, and showed that the octagonal cross-section has better characteristics from the point of view of vehicle crashworthiness under oblique load conditions. Qi et al.[22] provided a comparative study on the crashworthiness of four types of square tubes, namely single-cell straight, single-cell tapered, muti-cell straight and muti-cell taper, under oblique loading. It is found that the multi-cell tapered tube has the best crashworthiness performance under oblique impact regarding both specific energy absorption (SEA) and peak crushing force (PCF). However, the specific energy of multi-cell tapered tube still drops over the transition region by $53 \%$.

In order to improve the efficiency of materials utilization, Sun et al.[23] proposed a square tube with functionally graded thickness (FGT) and they showed that the FGT tube is superior to its uniform thickness(UT) counterparts in overall crashing behaviors under axial impact. Baykasoglu and Cetin[24] also revealed that the FGT circular tube exhibited better axial crashworthiness than its UT counterparts. Li et al.[25] compared the crashworthiness of FGT, taper uniform thickness and straight uniform thickness tubes and found that the FGT circular tube demonstrated the highest capacity of withstanding the oblique loading.

To take the advantage of FGT material and muti-cell structures, this paper explores the crashworthiness of a novel octagonal multi-cell tube with functionally graded thickness (FGT) under multiple loading angles. First, finite element analysis is conducted to comparatively study the crashworthiness of the FGT tube and UT tube when subject to oblique impact. Second, the effect of thickness gradient exponent and thickness range on the crashworthiness of the FGT tube is then explored. Third, Radial Basis Function technology and genetic algorithm are used to optimize the thickness gradient exponent and thickness range, to improve the crashworthy performance under uncertain loading conditions. The results reveal that the optimal FGT tube provides a better crashworthiness performance then the UT tube and thus can be a efficient energy absorber for withstanding oblique loading. 


\section{Materials and methods}

\subsection{Finite element model}

Figure 1 shows the sketch of the multi-cell tube. Based on the design of the bus, the tube has a length $\mathrm{L}=310 \mathrm{~mm}$. The side length of outer tube $\mathrm{D}=60 \mathrm{~mm}$, which is two times of the inner tube side length $\mathrm{d}$. The thicknesses for inner, outer and blade plates are the same and varies according to a power-law function along the axial direction of the tube as[23-27]:

$$
t(x)=t_{\text {top }}+\left(t_{\text {bot }}-t_{\text {top }}\right)\left(\frac{x}{L}\right)^{n}
$$

where $t_{\text {top }}$ and $t_{\text {bot }}$ are the thickness at the impact surface and the end surface, respectively. $x$ is the distance from the impact surface, $\mathrm{L}$ the length of the tube and $\mathrm{n}$ the gradient exponent which controls the variation of the thickness. The gradient function changes from convex to concave while the value of $\mathrm{n}$ changes from less than 1 to greater than 1 , as shown in Fig. 2.

To reveal the advantage of the FGT tubes for crashworthiness, the corresponding UT tubes are defined with the same weight as the FGT tubes. The equivalent thickness of the UT can be calculated by the following equation:

$$
t_{\text {avg }}=\frac{1}{N_{s}} \sum_{i=1}^{N_{s}} t_{i}
$$

where $N_{s}$ denotes the total number of layers of the FGF and $t_{i}$ is the thickness of the ith layer of the FGT. Therefore, the equivalent thickness of the UT solely depends on the gradient exponent sign $\mathrm{n}$ of the FGT.

Finite element models were built for the FGT and UT tubes based on their geometry, as shown in Fig. 3. The walls of tube were modeled with four-node Belytschko-Tsay thin shell element, which had 3 integration points through the thickness. The element size was $2 * 2 \mathrm{~mm}$. The tube material was the aluminum alloy 6061 and modeled with MAT_123 (MAT_MODIFIED_PIECE-WISE_LINEAR_PLASTICITY) in LS-DYNA (Version 971, LSTC, Livermore, CA, USA). The material constants are as follows: Density $\rho=2710 \mathrm{~kg} / \mathrm{m} 3$, Young's modulus E=68.9 GPa, Poisson's ratio $v=0.3$, yield strength $\sigma y=84.6 \mathrm{MPa}$. Effective stress-strain curve is shown in Figure 4. Since aluminum alloy is not sensitive to strain 
rate [16], the rate effect was not considered in the modeling. The tube impact onto the rigidwall at an initial velocity of

$50 \mathrm{~km} / \mathrm{h}$; while an additional mass block of $600 \mathrm{~kg}$ was attached to the bottom end to generate enough kinetic energy.

\subsection{Model validation}

When the gradient exponent $\mathrm{n}=0$, all the layers of the tube have the same thickness and the FGT tube become a UT tube; the model in this special case was validated by sled test $[23,24]$. The structure was mounted on a crash sled with the weight of $600 \mathrm{~kg}$ to test its crashworthiness. The setup is shown in Figure 5, where the initial impact velocity was 50 $\mathrm{km} / \mathrm{h}$. The model predicted deformation mode is compared with the specimen after test in Figure 6 . A progressive folding pattern can be observed in both physical and numerical models. The measured and predicted acceleration-time histories are shown in Figure 7. The peak occurred at about the same time and the curves exhibited the similar trend. Table 1 shows the relative errors between experiment and simulation. The crush distance of the tube in the test and simulation was $190 \mathrm{~mm}$ and $187 \mathrm{~mm}$, respectively, which is a discrepancy of $1.6 \%$. The measured and predicted acceleration-time histories are shown in Figure 7. The measured and calculated peak accelerations were $34.2 \mathrm{~g}$ and $31.7 \mathrm{~g}$, respectively.

The difference was $7.3 \%$. The average acceleration $\left(\bar{a}=\frac{1}{t} \int_{0}^{t} a(t) d t\right)$ was $22.8 \mathrm{~g}$ in the tests and $23 \mathrm{~g}$ in the simulations, respectively. The discrepancy was as low as $0.9 \%$. The reasonable agreement indicates that the numerical model was sufficiently validated and can be used in the further studies.

\subsection{Crashworthiness Index}

Generally, the parameters quantifying the structural crashworthiness mainly include total energy absorption $(E)$, specific energy $(S E A)$, average crush force $\left(F_{\text {avg }}\right)$, and Initial peak crush force $(I P F)[28]$. The total energy $E$ is the amount of energy absorption during the plastic deformation. It can be calculated by integrating the force-deflection curve as:

$$
E=\int_{0}^{d} F(x) d x
$$

where $d$ is the effective deflection and $F(x)$ is the transient force.

Specific energy is defined as the energy dissipation per unit mass. It is expressed in the form[29]:

$$
S E A=\frac{E}{M}
$$

with $M$ being the structural mass.

It has been reported that under the oblique impact, the impact angle has significant influence on SEA[22]. Here, we 
define the specific energy under the oblique loading as[22]:

$$
S E A_{\alpha}=\sum_{i=1}^{n} S E A^{\alpha i} w^{\alpha i}
$$

where $S E A^{\alpha i}$ is the specific energy at a certain impact angle $\alpha i(i=1,2, \cdots, n) ; w^{\alpha i}$ is the weighting factor, which is related to the probability of the impact at a certain angle. The sum of the weighting factors:

$$
\sum_{i=1}^{n} w^{\alpha i}=1 \quad w^{\alpha i} \geq 0
$$

Initial peak crush force is another important criterion for crashworthy assessment. It is defined as the peak value of initial spike on the force-deflection curve. In the case of oblique impact, the initial peak crush force is written as[22]:

$$
I P F_{\alpha}=\max \left(I P F_{\alpha i}, i=1,2, \cdots n\right)
$$

where $I P F_{a i}$ is the initial peak crush force at the impact angle of $\alpha i$.

\section{Results}

\subsection{Comparison of FGT and UT tubes}

Based on the aforementioned FE model, additional simulations were performed by changing the impact angle $\alpha$, which is defined as the angle between the top surface of tube and rigid plate, as shown in Fig. 1 ; the variation range $0^{\circ} \leq \alpha \leq 30^{\circ}$ is considered here.

The effects of loading angle on the SEA of FGT tube and UT tube are calculated at the same crushing displacement $225 \mathrm{~mm}(72 \%$ of the initial length), shown in Fig.8. It is seen that the SEA decrease with the increase in loading angle and three regions arise from the relationship between the SEA and loading angle: (1) a region where progressive crashing dominates (progressive region); (2) a region where progressive crashing transits to global buckling (transition region); and (3) a region where global buckling dominates (global buckling region). Similar phenomenon for the oblique loading has been reported by other researchers in literature[20, 22, 25]. From the detailed observation, the incident angle range of transition region is $\left(13^{\circ}, 15^{\circ}\right)$ for the UT tube and $\left(20^{\circ}, 23^{\circ}\right)$ for the FGT tube, respectively. If the loading angle is 
smaller than $13^{\circ}$, the FGT and UT tubes all fold progressively and generate similar SEA. However, with the increase of the loading angle, the FGT tube gradually demonstrate its advantages. When the loading angle varies from $13^{\circ}$ to $15^{\circ}$, the UT tube folds with the mixed mode of progressive crashing and global bending while the FGT tube folds progressively; the variation becomes bigger when the loading angle increase and the FGT tube exhibits $38.3 \%$ higher SEA than the UT tube at the loading angle of $15^{\circ}$. As the loading angle increase from $15^{\circ}$ to $20^{\circ}$, the UT tube folds with the global bending mode while the FGT tube still folds progressively; the SEA of FGT tube is $42.6 \%$ higher on average than UT tube in this region. The FGT tube then goes into transition region and global buckling region in succession, but it still bear higher SEA than that of the UT tube. In summary, the energy absorption is more superior for the FGT tube when the loading angle exceeds the lower bound of the transition range of the UT tube.

It is also important to assess the IPF which reflects the severity of collision and is highly related to the occupant injury. The maximum IPF always occurs under pure axial impact[22, 29-32]. Fig.9 depicts the force-displacement curves for both the FGT and UT tubes at the loading angle of $0^{\circ}$. FGT tube has relatively lower IPF than the UT counterpart, implying that it has better crashing safety in a low speed impact which is more common in reality[33, 34].

From the results shown above, the FGT tube shows a better performance in both SEA and IPF under oblique impact loading, compared with the UT counterparts. The FGT tube is thus selected as a suitable energy-absorbing structure for further analysis.

\subsection{Parametric analyses of FGT tubes}

Previous studies on square tube and circular tube with graded thickness demonstrated that the wall thickness gradient exponent $\mathrm{n}$ and thickness range $\triangle t$ has a great effect on the crashworthiness under axial impact[24, 35]. In this section, the effect of wall thickness gradient exponent $\mathrm{n}$ and thickness range $\triangle t$ on the crashworthiness of the octagonal multi-cell tube with functionally graded thickness under oblique impact is investigated. A baseline tube is set here with $\mathrm{T}_{\text {bot }}=3.0 \mathrm{~mm}, \quad \mathrm{~T}_{\text {top }}=1.2 \mathrm{~mm}$ and $\mathrm{n}=1$ for the parametric analyses. The gradient exponent $\mathrm{n}$ and thickness range $\triangle t$ of FGT 
tube are assumed as independent parameters in the following study.

\subsubsection{Effect of thickness gradient}

The effect of wall thickness gradient exponent $\mathrm{n}$ on SEA and IPF under multiple loading angles is shown in Fig.10 and

Fig.11, respectively. It can be seen in Fig.10 that, as $\mathrm{n}$ increases, SEA decreases at the loading angles of $0^{\circ}$ and $10^{\circ}$, while increases to a maximum and then decreases at the loading angles of $20^{\circ}$ and $30^{\circ}$. As shown in Fig.11, IPF decreases with $\mathrm{n}$ increasing from $0.2-1$ and changes little with $\mathrm{n}$ increasing from 1-10. One may conclude that the wall thickness gradient exponent has little effect on IPF when its value exceeds 2.

\subsubsection{Effect of thickness range}

Keeping $\mathrm{T}_{\mathrm{bot}}=3 \mathrm{~mm}$ and $n=1$ unchanged, the effect of $\triangle t\left(\triangle t=\mathrm{T}_{\mathrm{bot}}-\mathrm{T}_{\text {top }}\right)$ on SEA and IPF under multiple loading angles is studied by changing the value of $\mathrm{T}_{\text {top }}$, as illustrated in Fig.12 and Fig.13, respectively. Fig.12 shows that, as $\triangle t$ decreases, SEA increases at the loading angles of $0^{\circ}$ and $10^{\circ}$, while increases to a maximum and then decreases at the loading angles of $20^{\circ}$ and $30^{\circ}$. It can be found in Fig.13 that IPF increases as $\triangle t$ decreases at all loading angles.

Above analyses show that the wall thickness gradient exponent $\mathrm{n}$ and thickness range $\triangle t$ are both the important parameters that affect the SEA and IPF characteristic. Therefore, the FGT tube will be optimized with respect to these two variables in the next section.

\section{Optimization}

\subsection{Design methology}

In the optimal design of crashworthy structure, SEA should be one of the design objectives. On the other hand, it is desired to limit the peak crush force to a certain level. Hence, IPF is another design objective which should be minimized. In this study, $S E A_{\alpha}$ and $I P F_{\alpha}$ are chosen as two objective functions to improve overall crashworthiness in different loading angles[22].According to literature[27, 36], three design cases: namely(1)Case1: $w^{0^{\circ}}=w^{10^{\circ}}=w^{20^{\circ}}=w^{30^{\circ}}=0.25 ;(2)$ Case 2: $w^{0^{\circ}}=0.4, w^{10^{\circ}}=0.3, w^{20^{\circ}}=0.2$ and $w^{30^{\circ}}=0.1$; (3) Case $3: w^{0^{\circ}}=0.1, w^{10^{\circ}}=0.2, w^{20^{\circ}}=0.3$ and $w^{30^{\circ}}=0.4$, are considered 
to study the effect of weighting factors. This multi-objective optimization problem can be formulated as:

$$
\begin{cases}\max & \left\{S E A_{\alpha}(n, \Delta t),-I P F_{\alpha}(n, \Delta t)\right\} \\ \text { s.t. } & n^{L} \leq n \leq n^{U} \\ & \Delta t^{L} \leq \Delta t \leq \Delta t^{U}\end{cases}
$$

where $S E A_{\alpha}$ and $I P F_{\alpha}$ are respectively calculated by Eq.(5) and Eq.(7); $n^{L}$ and $n^{U}$ are the lower and upper limits of the gradient exponent n, $\triangle t^{L}$ and $\triangle t^{U}$ the lower and upper bounds for $\triangle t$, respectively. In the following optimization study, $n^{L}=0, n^{U}=10, \triangle t^{L}=0 \mathrm{~mm}$ and $\triangle t^{U}=2.2 \mathrm{~mm}$ are used.

Generally, meta model are often used for structural crashworthiness optimization since the complexity of deriving the objective functions. The common approaches for meta model building include RBF(radial basis function), RSM (Response Surface Method), MLS (Moving Least Square Method), Kriging and Forward feedback neural network[29]. Since RBF shows very good accuracy in the nonlinear impact problems [27, 37, 38], this approach is employed in the present study. The mathematical form of RBF can be formulated as:

$$
\hat{y}(x)=\sum_{i=1}^{n} \lambda_{i} \phi\left(r\left(\mathrm{x}_{i}, \mathrm{x}\right)\right)
$$

where $\mathrm{X}_{i}$ is the vector of design variables at the $i$ th design point, $\lambda_{i}$ is the coefficient of the linear combination of $n$ neurons and $\phi$ is a basis function as follows:

$$
\phi\left(r\left(\mathrm{x}_{i}, \mathrm{x}\right)\right)=\sqrt{\left\|\mathrm{x}-\mathrm{x}_{i}\right\|^{2}+c^{2}}
$$

where $\left\|\mathrm{x}-\mathrm{x}_{i}\right\|$ is the Euclidean distance, $c$ is equal to 1 in this paper.

To construct the RBF, the full factorial method is adopted for its ability to generate well-distributed sample points. To sample the design space, the gradient exponent $\mathrm{n}$ is selected as $0.05,0.2,0.4,0.6,0.8,1,2,4,6,8,10$, while $\triangle t$ is selected as $0.2 \mathrm{~mm}, 0.4 \mathrm{~mm}, 0.6 \mathrm{~mm}, 0.8 \mathrm{~mm}, 1.0 \mathrm{~mm}, 1.2 \mathrm{~mm}, 1.4 \mathrm{~mm}, 1.6 \mathrm{~mm}, 1.8 \mathrm{~mm}, 2.0 \mathrm{~mm}, 2.2 \mathrm{~mm}$. As a result, 121 sample points for each loading angle are obtained by using the full factorial design method. In addition, the loading angle varies from $0^{\circ}$ to $30^{\circ}$ with evenly interval of $10^{\circ}$. In total, 484 FEA runs are required, through which the values of IPF and SEA of the FGT tubes are evaluated. 
The results of optimization are highly dependent on the accuracy of Meta model. Therefore, it is necessary to check the Meta model before optimization takes place. Selecting four extra random validation points from the design space at each design case to evaluate the fitting accuracies of the RBF. Here, we use a relative error $R_{e}$ to evaluate the performance of the RBF, $R_{e}$ is defined as

$$
R_{e}=\left|\frac{f_{1}(x)-f_{2}(x)}{f_{1}(x)}\right|
$$

where $f_{1}(x)$ is simulation result, $f_{2}(x)$ is prediction based on the Meta model. From Table 2, it can be found that the relative errors in terms of both design objectives $S E A_{\alpha}$ and $I P F_{\alpha}$ are within $4 \%$. This indicates that the accuracy of meta model is sufficiently accurate for the following optimization.

\subsection{Optimization results}

NAGA- II algorithm is employed to seek the Pareto fronts of the multi-objective optimization problem as expressed by Eq.(8). NSGA II is a multiobjective genetic algorithm based on a fast nondominated sorting principle. The non-dominated set can be obtained by using the elitist non-dominated sorting along with sorting the crowding distance.

The non-dominated front is obtained after each generation. Finally, the non-dominated set is obtained with convergence of the iteration to form the Pareto fronts. This algorithm has proven rather effective for solving crashworthiness design problems[39, 40]. The optimizing parameters with population size $=12$, number of generations $=50$, crossover probability $=0.9$, mutation distribution index $=20.0$ and crossover distribution index $=10$ are selected. The Pareto fronts of the FGT tube for three design cases are generated and they are shown in Figure 14. It can be seen that $S E A_{\alpha}$ and $-I P F_{\alpha}$ are negatively related for each design case. In other words, increasing $S E A_{\alpha}$ will result in a lower $-I P F_{\alpha}$, and vice versa. In addition, Pareto fronts in the three cases have different distributions, the values of $S E A_{\alpha}$ in Case 2 are highest under a given $I P F_{\alpha}$. This is due to the fact that in Case 2, the weighting factor at the low $\alpha$ value is higher, and SEA is higher at low angle. It can be concluded that weighting factor has a great effect on the structural response of the tube. When designing a crashworthy structure, the weighting factors should be determined based on the statistical data. 
If the $I P F_{\alpha}$ need to be constrained under a value of $110 \mathrm{kN}$, the Pareto points corresponding to the optimal designs are marked as solid symbols and their detailed design parameters are listed in Table 3 . The FE models of these optimal designs are then established to compare with the meta model. As can be seen in Table 3, the errors are all less than $2 \%$, meaning that the optimized results obtained by RBF model have enough accuracy.

To further reveal the superiority of optimal FGT tube in crashworthiness, the UT tube is optimized and then compared with the optimized FGT tube. As illustrated in Fig.15, the pareto fronts of the FGT tubes are much predominant than those of the UT tubes, indicating that the FGT tube has the ability to increase $S E A_{\alpha}$ and decrease $I P F_{\alpha}$ simultaneously. For the three design cases, when $I P F_{\alpha}$ is constrained under $110 \mathrm{kN}, S E A_{\alpha}$ of the FGT tubes are respectively $50.63 \%, 45.11 \%$ and $54.21 \%$ higher than that of the UT tubes. Therefore, the optimized FGT tube has better crashworthiness than the optimized UT tube and can be a promising energy absorber.

\section{Conclusions}

This paper reports a computational study on the effect of impact angle and geometric parameters on the crashworthiness of a novel octagonal multi-cell tube with functionally graded thickness. Based on the numerical model, a multi-objective optimization was further carried out with weighting factors to improve the structural performance at the various loading orientations. The results show that(1) the progressive region of the FGT tube is wider than that of the counterpart UT tube; the energy absorption is more superior for the FGT tube when the loading angle exceeds the lower bound of the transition range of the UT tube; the FGT tube also has lower IPF $\alpha$ than the UT tube. (2) thickness gradient exponent and thickness range have significant effect on crashworthiness analysis and design. (3) Weighting factors dominate the distribution of Pareto fronts, and their values can be determined according to specific loading conditions and design requirements.

The crashworthiness of the optimized FGT tube is superior than the optimized UT tube; when IPF $\alpha$ is constrained under $110 \mathrm{kN}$, SEA $\alpha$ of the FGT tubes are respectively $50.63 \%, 45.11 \%$ and $54.21 \%$ higher than that of the UT tubes for the three design cases. 


\section{Acknowledgments}

The authors would like to thank the financial support from National Science Foundation (No. 51475153) and National

Science Foundation for Young Researchers (No. 51405148). The Project was also supported by the Science Fund of State

Key Laboratory of Advanced Design and Manufacturing for Vehicle Body (No. 3135001).

\section{References}

[1] J. Alexander, An approximate analysis of the collapse of thin cylindrical shells under axial loading. The Quarterly Journal of Mechanics and Applied Mathematics 1960;13:10-15.

[2] W. Abramowicz, N. Jones, Dynamic progressive buckling of circular and square tubes. International Journal of Impact Engineering 1986;4:243-270.

[3] S.R. Guillow, G. Lu, R.H. Grzebieta, Quasi-static axial compression of thin-walled circular aluminium tubes. International Journal of Mechanical Sciences 2001;43:2103-2123.

[4] X. Huang, G. Lu, T.X. Yu, On the axial splitting and curling of circular metal tubes. International Journal of Mechanical Sciences 2002;44:2369-2391.

[5] A.W. Wierzbicki T, On the Crushing Mechanics of Thin-Walled Structures. Journal of Applied mechanics 1983;50:727-734.

[6] W. Abramowicz, N. Jones, Dynamic axial crushing of square tubes. International Journal of Impact Engineering 1984;2:179-208.

[7] A.G. Mamalis, D.E. Manolakos, A.K. Baldoukas, G.L. Viegelahn, Energy dissipation and associated failure modes when axially loading polygonal thin-walled cylinders. Thin-Walled Structures 1991;12:17-34.

[8] A.G. Mamalis, D.E. Manolakos, M.B. Ioannidis, P.K. Kostazos, C. Dimitriou, Finite element simulation of the axial collapse of metallic thin-walled tubes with octagonal cross-section. Thin-Walled Structures 2003;41:891-900.

[9] G.M. Nagel, D.P. Thambiratnam, Dynamic simulation and energy absorption of tapered thin-walled tubes under oblique impact loading. International Journal of Impact Engineering 2006;32:1595-1620.

[10] Z. Fan, G. Lu, K. Liu, Quasi-static axial compression of thin-walled tubes with different cross-sectional shapes. Engineering Structures 2013;55:80-89.

[11] A. Rossi, Z. Fawaz, K. Behdinan, Numerical simulation of the axial collapse of thin-walled polygonal section tubes. Thin-Walled Structures 2005;43:1646-1661.

[12] Z. Zhang, S. Liu, Crashworthiness of dynamical axial crushing of polygonal thin-walled tubes, in: SAE-China 
congress proceedings, China Machine Press, Beijing, 2007, pp. 437-443.

[13] T. Wierzbicki, N. Jones, Structural failure, Wiley-Interscience, 1989.

[14] X. Zhang, G. Cheng, H. Zhang, Theoretical prediction and numerical simulation of multi-cell square thin-walled structures. Thin-Walled Structures 2006;44:1185-1191.

[15] A. Jusuf, T. Dirgantara, L. Gunawan, I.S. Putra, Crashworthiness analysis of multi-cell prismatic structures. International Journal of Impact Engineering 2015;78:34-50.

[16] A.A. Nia, M. Parsapour, Comparative analysis of energy absorption capacity of simple and multi-cell thin-walled tubes with triangular, square, hexagonal and octagonal sections. Thin-Walled Structures 2014;74:155-165.

[17] T. Zhiliang, L. Shutian, Z. Zonghua, Analysis of energy absorption characteristics of cylindrical multi-cell columns. Thin-Walled Structures 2013;62:75-84.

[18] T. TrongNhan, H. Shujuan, H. Xu, C. MinhQuang, Crushing analysis and numerical optimization of angle element structures under axial impact loading. Compos. Struct. 2015;119:422-435.

[19] H. Shujuan, L. Qing, L. Shuyao, Y. Xujing, L. Wei, Multiobjective optimization of multi-cell sections for the crashworthiness design. International Journal of Impact Engineering 2008;35:1355-1367.

[20] D.C. Han, S.H. Park, Collapse behavior of square thin-walled columns subjected to oblique loads. Thin-Walled Structures 1999;35:167-184.

[21] P. Hosseini-Tehrani, S. Pirmohammad, Collapse study of thin-walled polygonal section columns subjected to oblique loads. Proc. Inst. Mech. Eng. Part D-J. Automob. Eng. 2007;221:801-810.

[22] C. Qi, S. Yang, F. Dong, Crushing analysis and multiobjective crashworthiness optimization of tapered square tubes under oblique impact loading. Thin-Walled Structures 2012;59:103-119.

[23] S. Guangyong, X. Fengxiang, L. Guangyao, L. Qing, Crashing analysis and multiobjective optimization for thin-walled structures with functionally graded thickness. International Journal of Impact Engineering 2014;64:62-74.

[24] C. Baykasoglu, M.T. Cetin, Energy absorption of circular aluminium tubes with functionally graded thickness under axial impact loading. International Journal of Crashworthiness 2015;20:95-106.

[25] G. Li, F. Xu, G. Sun, Q. Li, A comparative study on thin-walled structures with functionally graded thickness (FGT) and tapered tubes withstanding oblique impact loading. International Journal of Impact Engineering 2015;77:68-83.

[26] M.S. Attia, S.A. Meguid, H. Nouraei, Nonlinear finite element analysis of the crush behaviour of functionally graded foam-filled columns. Finite Elements in Analysis and Design 2012;61:50-59.

[27] G. Li, Z. Zhang, G. Sun, F. Xu, X. Huang, Crushing analysis and multiobjective optimization for functionally graded foam-filled tubes under multiple load cases. International Journal of Mechanical Sciences 2014;89:439-452. 
[28] G. Lu, T. Yu, Energy absorption of structures and materials, Elsevier, 2003.

[29] Y. Zhang, G. Sun, X. Xu, G. Li, Q. Li, Multiobjective crashworthiness optimization of hollow and conical tubes for multiple load cases. Thin-Walled Structures 2014;82:331-342.

[30] S. Yang, C. Qi, Multiobjective optimization for empty and foam-filled square columns under oblique impact loading. International Journal of Impact Engineering 2013;54:177-191.

[31] Z. Ahmad, D. Thambiratnam, A. Tan, Dynamic energy absorption characteristics of foam-filled conical tubes under oblique impact loading. International Journal of Impact Engineering 2010;37:475-488.

[32] G. Li, Z. Zhang, G. Sun, X. Huang, Q. Li, Comparison of functionally-graded structures under multiple loading angles. Thin-Walled Structures 2015;94:334-347.

[33] Z. Ahmad, D.P. Thambiratnam, Dynamic computer simulation and energy absorption of foam-filled conical tubes under axial impact loading. Computers \& Structures 2009;87:186-197.

[34] L. Mirfendereski, M. Salimi, S. Ziaei-Rad, Parametric study and numerical analysis of empty and foam-filled thin-walled tubes under static and dynamic loadings. International Journal of Mechanical Sciences 2008;50:1042-1057.

[35] G. Zheng, S. Wu, G. Sun, G. Li, Q. Li, Crushing analysis of foam-filled single and bitubal polygonal thin-walled tubes. International Journal of Mechanical Sciences 2014;87:226-240.

[36] Z. Bai, J. Liu, F. Zhu, F. Wang, B. Jiang, Optimal design of a crashworthy octagonal thin-walled sandwich tube under oblique loading. International Journal of Crashworthiness 2015;20:401-411.

[37] G. Sun, G. Li, Z. Gong, G. He, Q. Li, Radial basis functional model for multi-objective sheet metal forming optimization. Engineering Optimization 2011;43:1351-1366.

[38] H. Yin, G. Wen, Z. Liu, Q. Qing, Crashworthiness optimization design for foam-filled multi-cell thin-walled structures. Thin-Walled Structures 2014;75:8-17.

[39] Y. Zhang, G. Sun, G. Li, Z. Luo, Q. Li, Optimization of foam-filled bitubal structures for crashworthiness criteria. Materials \& Design 2012;38:99-109.

[40] X. Liao, Q. Li, X. Yang, W. Zhang, W. Li, Multiobjective optimization for crash safety design of vehicles using stepwise regression model. Structural and Multidisciplinary Optimization 2008;35:561-569. 
Captions to the illustrations

Fig.1. Geometrical configuration and boundary condition of tubes under oblique impact.

Fig.2. Variation in thickness versus distance ( $0=$ bottom end surface, $1=$ impact surface).

Fig.3. Illustration of finite element model: (a) FGF tube, (b) UT tube.

Fig.4. The effective flow stress-plastic strain curve of aluminum 6061.

Fig.5. The specimen on the test sled.

Fig.6. Deformation pattern of UT tube in sled test and simulation.

Fig.7. Longitudinal acceleration-time curves of experiment and simulation.

Fig.8. Effect of load angle on the SEA of FGT and UT structures.

Fig.9. The force-displacement responses for FGT and UT under axial impact.

Fig.10. Effects of thickness gradient exponent $n$ on SEA under oblique impact.

Fig.11. Effects of thickness gradient exponent $n$ on IPF under oblique impact.

Fig.12. Effects of thickness range $\triangle t$ on SEA under oblique impact.

Fig.13. Effects of thickness range $\triangle t$ on IPF under oblique impact.

Fig.14. Pareto fronts for FGT tube under three different design cases.

Fig.15. Pareto fronts for FGT and UT structures under three different design cases.(a) Case1,(b)Case 2,(c)Case 3. 


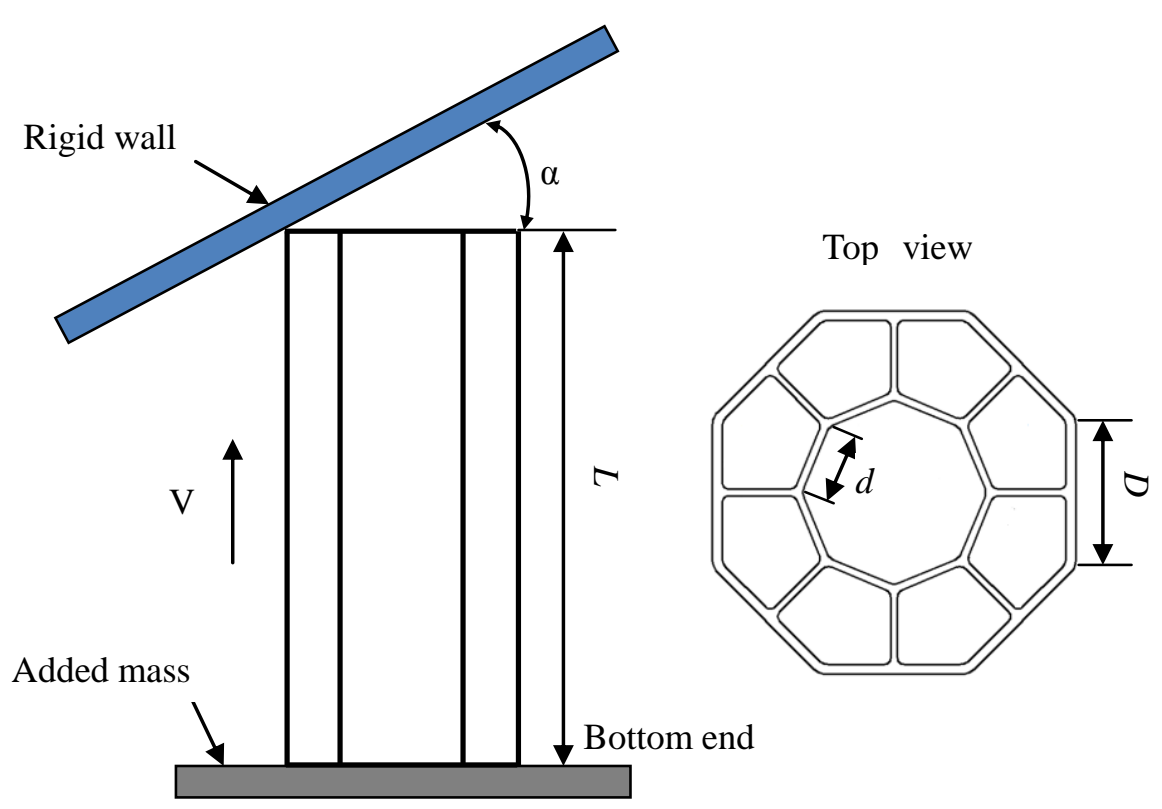

Fig.1. 


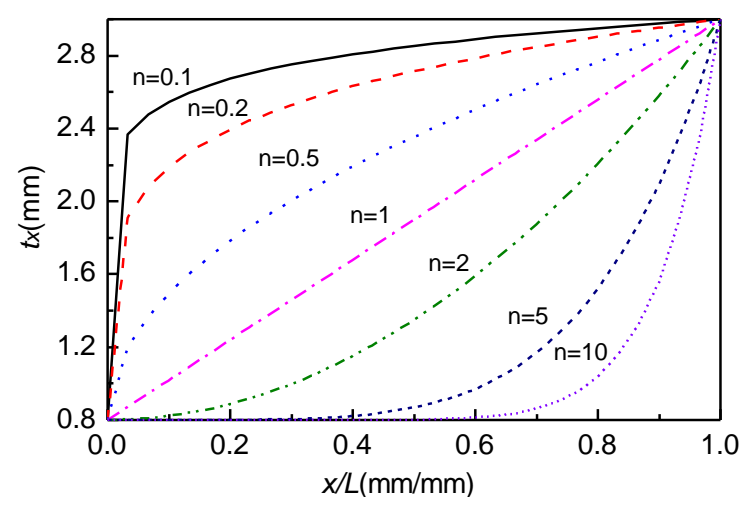

Fig.2. 
a

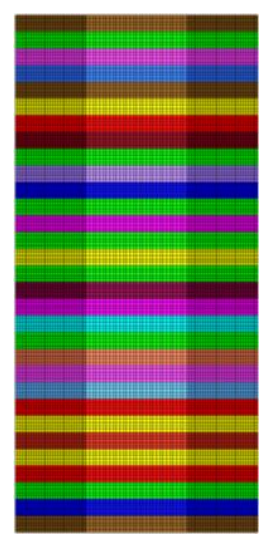

b

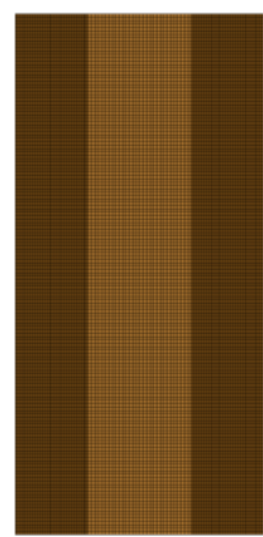

Fig.3. 


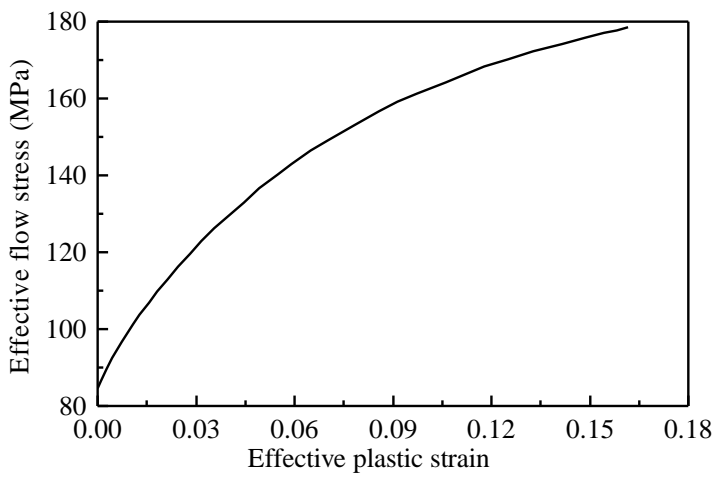

Fig.4. 


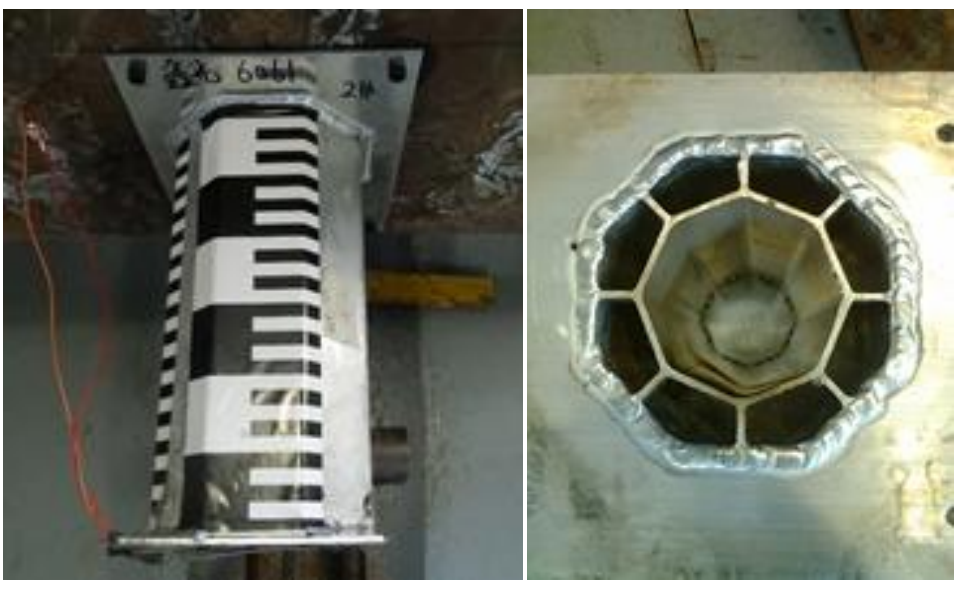

Fig.5. 


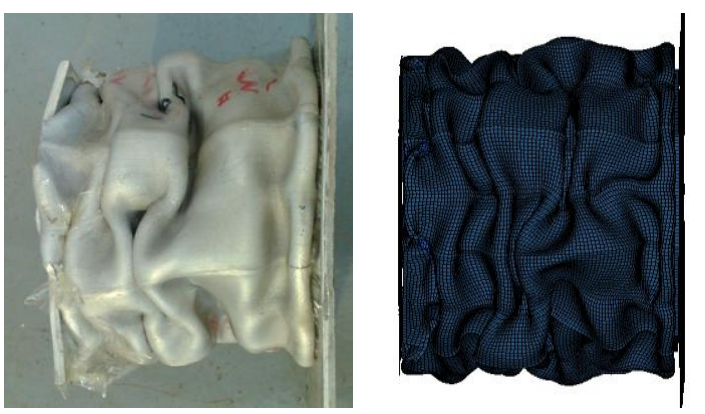

Fig.6. 


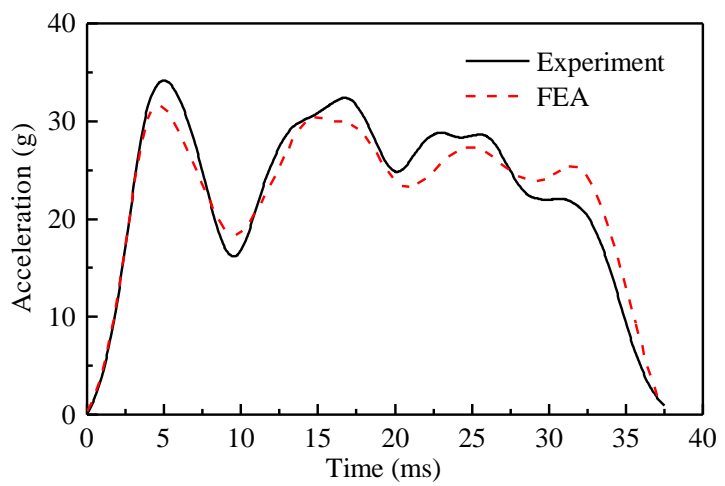

Fig.7. 


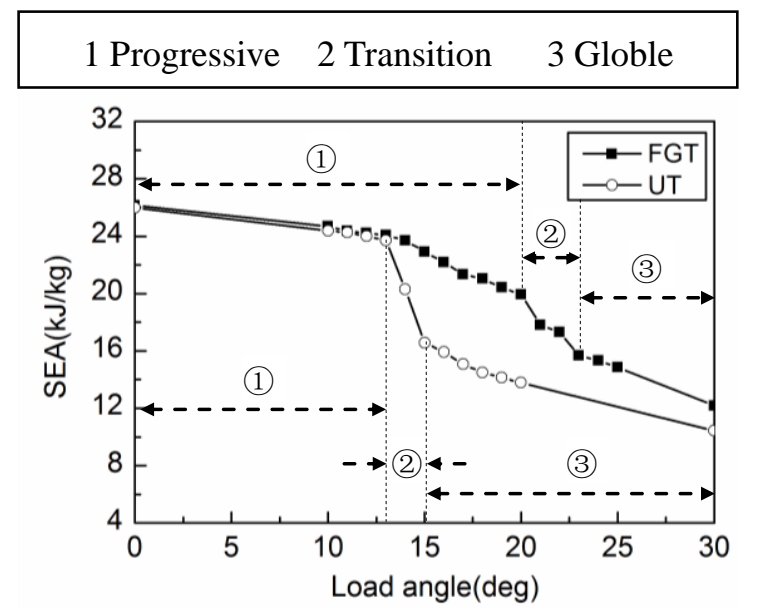

Fig.8. 


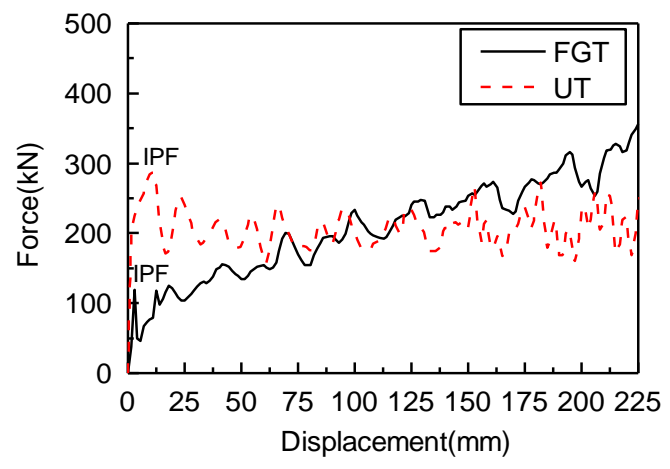

Fig.9. 


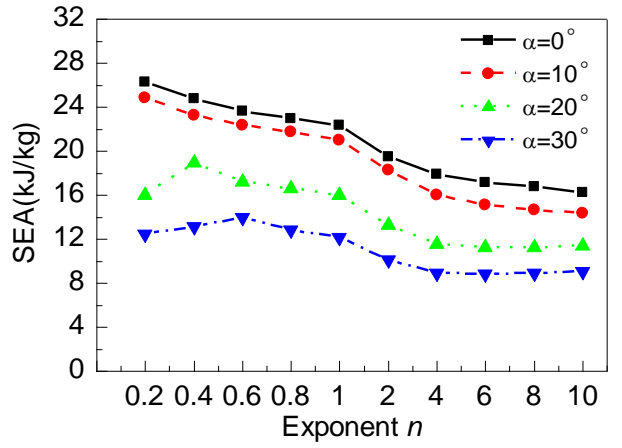

Fig.10. 


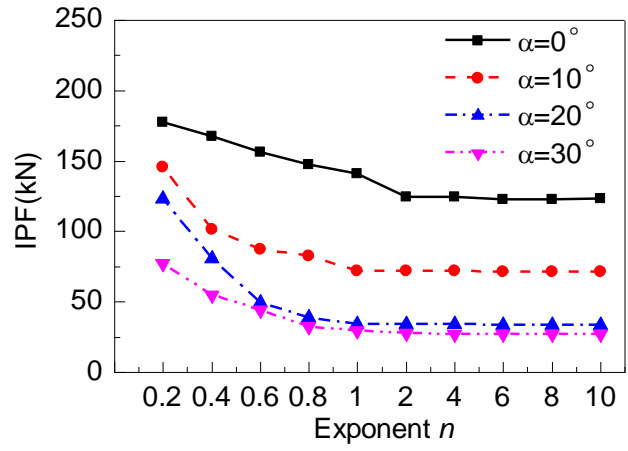

Fig.11. 


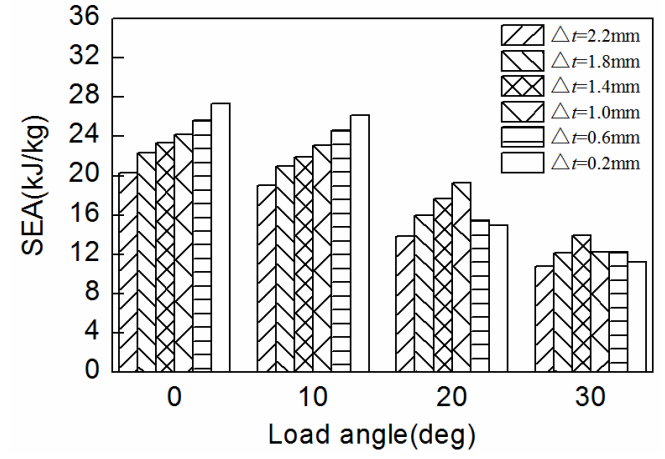

Fig.12. 


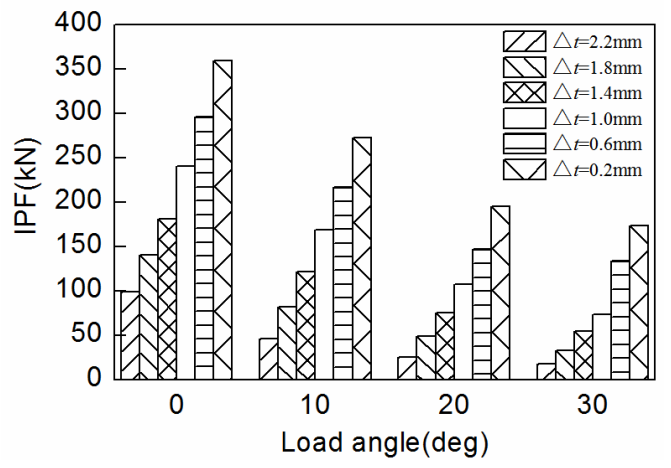

Fig.13. 


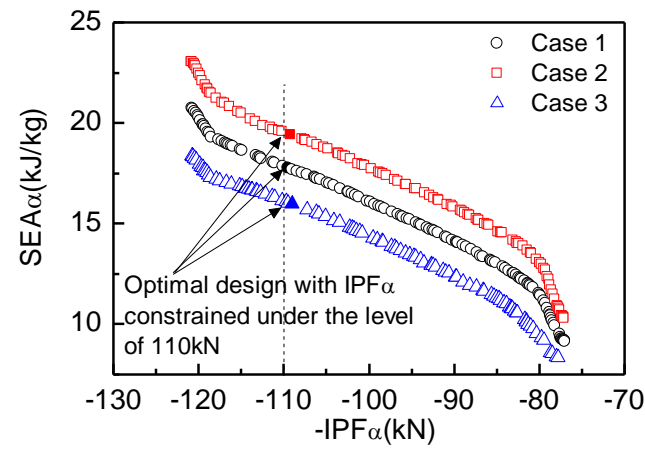

Fig.14. 


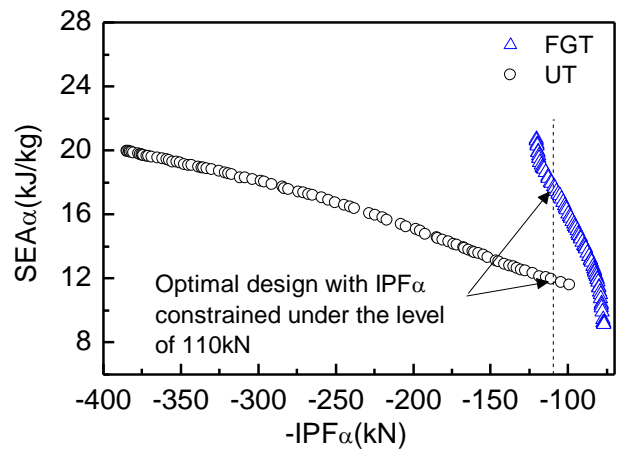

(a)

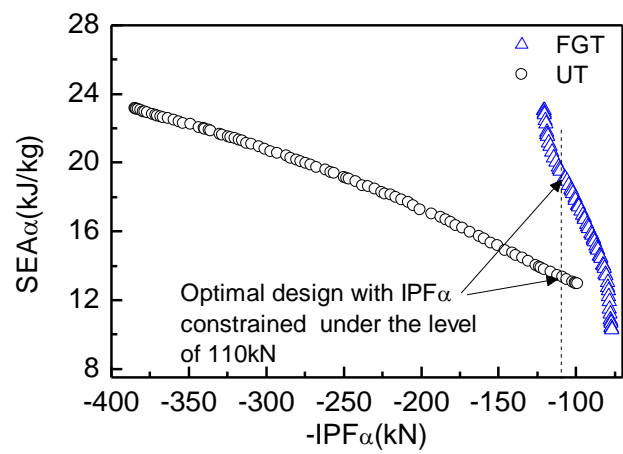

(b)

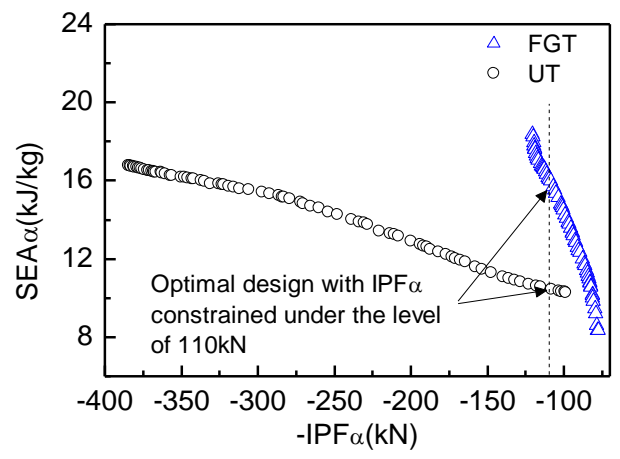

(c)

Fig.15. 
List of the tables

Table 1. Relative errors between experiment and simulation.

Table 2. Accuracies of RBF for three design cases.

Table 3. Optimal design parameters of FGT tube with $I P F_{\alpha}$ constrained under $110 \mathrm{kN}$. 
Table 1.

Crush distance (mm) Peak acceleration (g)

average acceleration $(\mathrm{g})$

\begin{tabular}{cccc}
\hline Experiment & 190 & 34.2 & 22.8 \\
Simulation & 187 & 31.7 & 23 \\
Relative errors & $1.6 \%$ & $7.3 \%$ & $0.9 \%$ \\
\hline
\end{tabular}


Table 2.

\begin{tabular}{lcccccc}
\hline & \multicolumn{3}{c}{$\boldsymbol{S E A _ { \alpha } : \operatorname { R e } ( \% )}$} & \multicolumn{3}{c}{$\boldsymbol{I P F _ { \alpha } : \operatorname { R e } ( \% )}$} \\
\cline { 2 - 6 } & Case1 & Case2 & Case3 & Case1 & Case2 & Case3 \\
\hline The sample point 1 & 2.33 & 3.14 & 3.01 & 2.20 & 3.23 & 1.83 \\
The sample point 2 & 2.20 & 1.25 & 2.71 & 3.29 & 2.40 & 3.22 \\
The sample point 3 & 1.37 & 3.16 & 2.68 & 2.33 & 3.19 & 3.03 \\
The sample point 4 & 3.42 & 2.74 & 2.44 & 3.16 & 3.34 & 2.87 \\
\hline
\end{tabular}


Table 3.

\begin{tabular}{|c|c|c|c|c|c|c|c|c|}
\hline \multirow{2}{*}{ Design Case } & \multirow{2}{*}{$n$} & \multirow{2}{*}{$\triangle t$} & \multicolumn{3}{|c|}{$S E A_{\alpha}(\mathrm{kJ} / \mathrm{kg})$} & \multicolumn{3}{|c|}{$I P F_{\alpha}(\mathrm{kN})$} \\
\hline & & & RBF model & FEA & Error(\%) & RBF model & FEA & Error $(\%)$ \\
\hline 1 & 0.6739 & 2.1998 & 17.848 & 17.639 & -1.184 & 109.972 & 111.263 & 1.160 \\
\hline 2 & 0.6941 & 2.1999 & 19.418 & 19.126 & -1.527 & 109.216 & 110.728 & 1.366 \\
\hline 3 & 0.6801 & 2.1992 & 16.112 & 16.238 & 0.776 & 109.787 & 111.159 & 1.234 \\
\hline
\end{tabular}

